\title{
Association of the HDL-c Level with HsCRP, IL-6, U-NAG, RBP and Cys-C in Type 2 Diabetes Mellitus, Hypertension, and Chronic Kidney Disease: An Epidemiological Survey
}

This article was published in the following Dove Press journal:

Diabetes, Metabolic Syndrome and Obesity: Targets and Therapy

\begin{abstract}
Xiaolin $\mathrm{Li}^{1,2}$
Ting $\mathrm{Su}^{3}$

Hua Xiao'

Peichun $\mathrm{Gao}^{4}$

Chongxiang Xiong'

Jinghua Liu (iD ${ }^{3}$

Hequn Zou'

'Department of Nephrology, The Third Affiliated Hospital, Southern Medical University, Guangzhou 510630, People's Republic of China; ${ }^{2}$ Department of Endocrinology, Hunan University of Medicine, Huaihua 418000, Hunan, People's Republic of China; ${ }^{3}$ Guangdong Provincial Key Laboratory of Proteomics, School of Basic Medical Sciences,

Southern Medical University, Guangzhou 510515, People's Republic of China; ${ }^{4}$ School of Public Health, Southern Medical University, Guangzhou 5I0080, People's Republic of China
\end{abstract}

Correspondence: Jinghua Liu Guangdong Provincial Key Laboratory of Proteomics, School of Basic Medical Sciences, Southern Medical University, Guangzhou 510515, People's Republic of China

Tel +862061648392

Fax +86206164823I

Email liujhua@smu.edu.cn

Hequn Zou

Department of Nephrology, The Third Affiliated Hospital, Southern Medical University, 183 Zhongshan Dadao, Tianhe District, Guangzhou 5I 0630, People's Republic of China

Tel +862062784393

$\mathrm{Fax}+862062784399$

Email hequnzou@hotmail.com

\begin{abstract}
Purpose: To explore the association between the anti-inflammatory and renal protective roles of high-density lipoprotein cholesterol (HDL-c) and its different levels in type 2 diabetes mellitus (T2D), hypertension (HTN), and chronic kidney disease (CKD) and to lay a theoretical basis for precise, maximum-benefit HDL-c-raising therapy for patients with these diseases.
\end{abstract}

Patients and Methods: A total of 2127 participants (195 with T2D, 618 with HTN, 162 with CKD, and 1152 controls) were selected and divided into four groups according to their baseline HDL-c level, namely, low HDL-c (L-HDL-c, $\leq 1.03 \mathrm{mmol} / \mathrm{L}$ ), medium HDL-c (MHDL-c, 1.04-1.55 mmol/L), high HDL-c (H-HDL-c, 1.56-2.05 mmol/L) and extremely high HDL-c (E-HDL-c, $\geq 2.06 \mathrm{mmol} / \mathrm{L}$ ). Serum and morning urine samples were collected to analyze the correlation between high-sensitivity C-reactive protein (HsCRP), interleukin-6 (IL-6), urine n-acetyl- $\beta$-d-glucosidase (U-NAG), retinol binding protein (RBP), and cystatin c (Cys-C) levels with the HDL-c levels.

Results: The HDL-c levels of patients with T2D, HTN and CKD were universally lower than those in the control group in both sexes $(p<0.05)$, while male patients also manifested a lower level of HDL-c than female patients. However, although they had lower values of the renal impairment index, female patients were found to have anomalously higher amounts of proinflammatory cytokines. In addition, the correlations between HsCRP and RBP levels and HDL-c levels were most significant in patients with HTN $(p<0.05)$, whereas in patients with T2D and CKD, such relevance was less significant.

Conclusion: Existence of substantial differences in HDL-c levels between different types of disease and sex highlighted that a higher HDL level does not always predict a better clinical outcome of patients. Moreover, we found that both HsCRP and RBP correlated negatively with HDL-c in HTN patients, indicating that monitoring HsCRP and RBP may serve as indicators for therapeutic efficacy of HDL-c-raising medications in HTN patients.

Keywords: high-density lipoprotein cholesterol, anti-inflammation, renal protection, sex difference, functional heterogeneity

\section{Introduction}

Dyslipidemia is involved in vascular complications in patients with type 2 diabetes mellitus (T2D), hypertension (HTN), and chronic kidney disease (CKD) ${ }^{1-3}$ Early studies have uncovered that increasing high-density lipoprotein cholesterol (HDL-c) levels is an effective way to prevent atherosclerosis. ${ }^{4}$ HDL particles have a variety of important 
functions that protect the vascular endothelium based on their anti-inflammatory and antioxidative effects. ${ }^{5}$ Raising HDL-c levels is a typical treatment strategy for these diseases.

However, the clinical results were not as satisfactory as expected. Recent large clinical trials have shown that increasing HDL-c levels with drugs did not decrease the risk of cardiovascular events. Lincoff et al found that despite a 31\% decrease in LDL-c and a significant 133\% increase in HDL-c, the incidence of major adverse cardiac events was not lower than that of the placebo group after 26 months of treatment. ${ }^{6}$ Ziyad Al-Aly et al tracked 192,683 male veterans in the US Veterans Database for nine years. After adjusting for a series of factors, they built a U-shaped correlation curve between HDLc level and kidney disease outcomes. ${ }^{7}$ Similarly, cohort studies discovered that an extremely high HDL-c level was associated with high mortality and morbidity. ${ }^{8-10}$ Furthermore, genomewide association studies (GWAS) have revealed that deficient HDL-c volume was not a determinant of cardiovascular disease $^{11,12}$ and that elevating HDL-c levels did not always contribute to attenuating atherosclerosis. ${ }^{13}$ All these emerged evidences have warned that a higher HDL-c level does not always benefit the clinical outcome of patients. In fact, it is unclear whether patients of different sexes or those with different diseases could get the equal benefit from the medication-maintained same HDL-c level. This study tried to probe the anti-inflammatory and renal protective role of HDL-c at different levels in three of the most common dyslipidemiaassociated diseases in public health, namely, T2D, HTN and $\mathrm{CKD}$, via large-scale sample detection and statistical analysis.

Involved in the processes of T2D, HTN and CKD, inflammation plays an important role in the genesis and development of atherosclerosis and renal injury. The realization of the advantageous effects of HDL depends largely on its anti-inflammatory function. ${ }^{14}$ There are many studies on the changes of high-sensitivity C-reactive protein (HsCRP), interleukin-6 (IL-6) and HDL-c in patients with T2D, HTN or CKD, but few studies have assessed the associations between HDL-c and HsCRP, IL-6 in these diseases, particularly the HDL-c levels. ${ }^{15-17}$ Moreover, the few studies paying attention to the correlation between early renal damage indicators and HDL-c levels have inspired us to investigate the complex interaction network in these diseases. Canonical predictors, including urine n-acetyl- $\beta$ d-glucosidase (U-NAG), retinol binding protein (RBP) and cystatin $\mathrm{C}$ (Cys-C), were then introduced to assess the degree of renal injury, especially in its early stage.

Overall, we conducted an epidemiological survey (1) to assess the differential level of HDL-c among patients with
T2D, HTN or CKD and healthy participants, (2) to investigate whether there is a sex difference, and (3) to study the correlation between HsCRP, IL-6, U-NAG, RBP, Cys$\mathrm{C}$ levels and HDL-c levels in those patients to provide a theoretical guide for clinical treatment targeting HDL-c.

\section{Patients and Methods}

This study is based on an epidemiological survey conducted in Wanchai town, Zhuhai city, China, from December 2017 to March 2018. A total of 3553 participants were randomly selected from 4 communities in Wanchai town. Among them, 2127 participants diagnosed with T2D, HTN or CKD without lipid-lowering therapy were selected as the research sample, and other healthy participants were taken as the control group. Detailed information on the grouping is shown in Figure 1. This study was conducted in accordance with the Declaration of Helsinki. The study protocol was approved by the ethics committee of the Third Affiliated Hospital of Southern Medical University, and all participants signed an informed consent form.

\section{Subjects}

Patients with T2D, HTN or CKD, aged 18 to 75, were selected as research subjects. The T2D group included patients whose fasting blood glucose was over $7.0 \mathrm{mmol} / \mathrm{L}$ or whose random blood glucose was over $11.1 \mathrm{mmol} / \mathrm{L}$, with self-reported physician diagnosis or current use of hypoglycemic drugs. ${ }^{18}$ HTN was defined as the use of oral antihypertensive drugs or systolic blood pressure exceeding $140 \mathrm{mmHg}$ and/or diastolic blood pressure exceeding $90 \mathrm{mmHg}$, measured in a sitting position on at least three different occasions. ${ }^{19} \mathrm{CKD}$ was defined as chronic structural and functional impairment of the kidney with an unexplained decrease in glomerular filtration rate $\left(\mathrm{GFR}<60 \mathrm{~mL} / \mathrm{min} \bullet 1.73 \mathrm{~m}^{2}\right)$ for more than 3 months. ${ }^{20}$ A simplified MDRD (Modification of Diet in Renal Disease) formula was used to calculate the estimated glomerular filtration rate (eGFR). ${ }^{21}$ The T2D group excluded patients with HTN or CKD, and the HTN group excluded patients with T2D or CKD, while the CKD group included all patients with CKD who may have T2D, HTN, or both diseases. Patients with infectious diseases, rheumatic and immune diseases, liver diseases, tumors, blood diseases or acute complications were excluded. At the same time, subjects treated with fibrates, cholesterol ester transfer protein (CETP) inhibitors, and statins were also excluded, as these drugs may increase HDL-c levels. The body mass index (BMI) was the body weight $(\mathrm{kg})$ divided by the squared height $(\mathrm{m})$. 


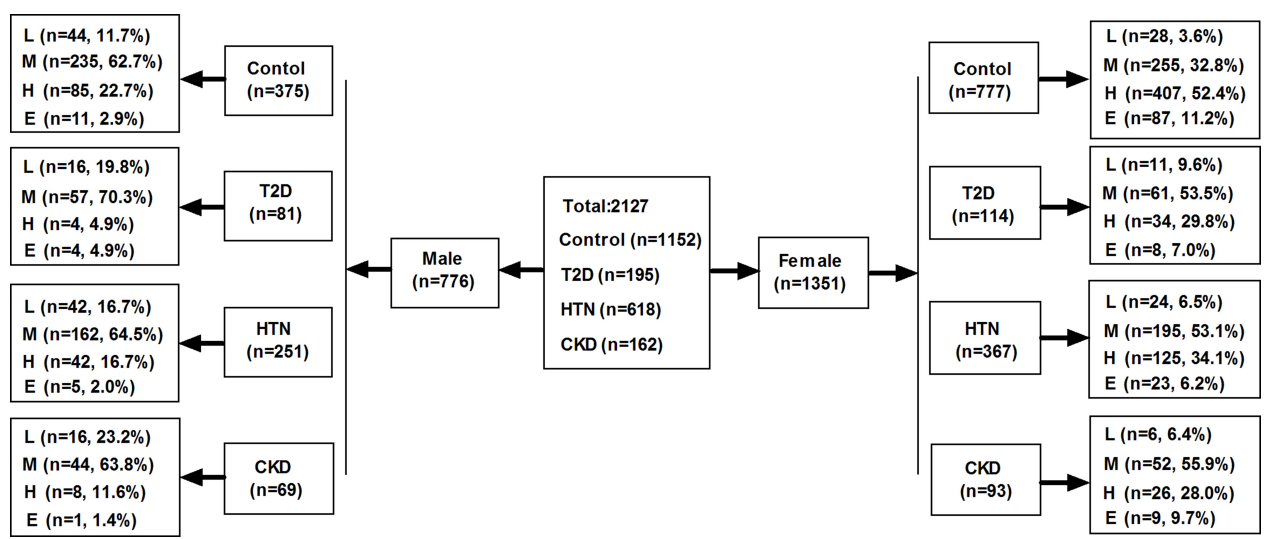

Figure I Flow chart of the participant distribution.

Abbreviations: HDL-c, high-density lipoprotein cholesterol; L, low HDL-c; M, medium HDL-c; H, high HDL-c; E, extremely high HDL-c; control, healthy participants; T2D, type 2 diabetes mellitus; HTN, hypertension; CKD, chronic kidney disease.

All participants were divided into four groups: low HDLc (L-HDL-c, $\leq 1.03 \mathrm{mmol} / \mathrm{L})$, medium HDL-c (M-HDL-c, $1.04-1.55 \mathrm{mmol} / \mathrm{L}$ ), high HDL-c (H-HDL-c, 1.56$2.05 \mathrm{mmol} / \mathrm{L}$ ) and extremely high HDL-c (E-HDL-c, $\geq 2.06 \mathrm{mmol} / \mathrm{L})^{22}$

\section{Specimen Collection}

Serum samples (after fasting for at least 10 hours overnight) and urine samples (the second urine after primary urine at $7 \mathrm{am}$ ) were collected and analyzed at the central laboratory of the Third Affiliated Hospital of Southern Medical University on the same day of collection. All specimens were stored at $4^{\circ} \mathrm{C}$ before analysis.

\section{Methods and Reagents}

Fasting blood glucose (FBG) was determined by the hexokinase method (Maccura Ltd., Chengdu, China); HDL-c was detected by the enzymatic method (Jingyuan Medical Apparatus and Instruments, Ltd., Shanghai, China); U-NAG was measured by the MPT substrate method (Huayuyikang Bioengineering Technology Ltd., Beijing, China); and Cys-C was evaluated by the latex immunoturbidimetric method (Gcell, Jiuqiang Biotechnology Ltd., Beijing, China). Immune turbidimetry (Beijia BIO-reagent Ltd., Shanghai, China) was applied to test RBP, and immune turbidimetry (Orion Diagnostics Stica Oy, Espoo, Finland) was utilized for HsCRP. IL-6 was determined by electrochemiluminescence (Roche Diagnostics GmbH, Mannheim, Germany).

\section{Statistical Analysis}

SPSS 22.0 software (International Business Machine, USA) was used to process the research data. The distribution of data was checked first. The results for continuous variables without a normal distribution are presented as the median (interquartile range, IQR), and those with a normal distribution are presented as the mean \pm standard deviation (SD). The classification data are presented as proportions or rates, and the Chi-squared test was used to compare the proportion of HDL-c level distribution between patients with T2D, HTN or CKD and the control group. The Mann-Whitney U-test was used to analyze the difference between two groups, and the Kruskal-Wallis test was applied to test the difference between multiple groups for the correlations between HsCRP, IL-6, U-NAG, RBP, Cys-C and HDL-c. Significant differences were expressed as $p<0.05$.

\section{Results}

The demographic and clinical characteristics of the overall study are presented in Table 1. All 2127 participants, 776 males and 1351 females, were divided into four groups. The incidence rates of T2D, HTN and CKD in Wanchai town of Zhuhai city are $9.2 \%, 29.1 \%$ and $7.6 \%$, respectively, which are similar to those of other regions in China $(8.9-10.4 \%, 25.2 \%$ and $8-16 \%) .{ }^{23-27}$

\section{Patients with T2D, HTN or CKD Had Significantly Lower Levels of HDL-c Than the Control Group}

Overall, the HDL-c levels of patients with T2D, HTN or CKD were significantly lower than those of the control group for both sexes ( $p<0.05$, Figure 2A, 2B, 2C). L-HDL$\mathrm{c}$ and M-HDL-c accounted for a larger proportion of HDL$\mathrm{c}$ in female patients with T2D, HTN or CKD and in male patients with T2D or CKD $(p<0.05$, Figure 2D, 2E, $2 \mathrm{~F})$. 
Table I Baseline Characteristics of the Subjects on the Basis of HDL-c

\begin{tabular}{|c|c|c|c|c|c|}
\hline Male & L-HDL-c & M-HDL-c & H-HDL-c & E-HDL-c & Total \\
\hline Number (\%) & $118(15.21)$ & $504(64.95)$ & $139(|7.9|)$ & $15(1.93)$ & 776 \\
\hline HDL-C (IQR) mmol/L) & $0.94(0.87,0.99)$ & $1.27(1.15,1.40)$ & I.7I (I.62,I.82) & $2.32(2.14,2.55)$ & $1.29(1.10,1.50)$ \\
\hline Age (IQR) (years) & $61(52,72)$ & $58(50,69)$ & $62(51,68)$ & $63(55,76)$ & $60(51,69)$ \\
\hline BMI (IQR) $\left(\mathrm{kg} / \mathrm{m}^{2}\right)$ & $25.6(23.7,27.5)$ & $25 . I(23.2,27.4)$ & $22.6(20.5,24.4)$ & $22.2(20.5,25.3)$ & $24.8(22.6,26.9)$ \\
\hline FBG (IQR) (mmol/L) & $5.4(4.8,6.4)$ & $5 . I(4.7,5.9)$ & $4.9(4.5,5.4)$ & $5.1(4.8,6.0)$ & $5.1(4.7,5.8)$ \\
\hline LDL (IQR) (mmol/L) & $2.6(1.9,3.2)$ & $3.2(2.7,3.8)$ & $3.2(2.5,3.7)$ & $3.2(2.1,4.3)$ & $3.1(2.5,3.7)$ \\
\hline TG (IQR) (mmol/L) & $2.6(1.5,3.8)$ & I.6(I.2,2.2) & $1.0(0.8,1.4)$ & $0.9(0.7,1.6)$ & I.6(I.I,2.2) \\
\hline $\mathrm{TC}(\mathrm{IQR})(\mathrm{mmol} / \mathrm{L})$ & $4.9(4.2,5.6)$ & $5.3(4.7,5.9)$ & $5.4(4.7,6.1)$ & 6.I $I(5.2,7.2)$ & $5.3(4.6,5.9)$ \\
\hline VLDL-c(IQR) (mmol/L) & I.2(0.7,I.7) & $0.7(0.5,1.0)$ & $0.5(0.4,0.6)$ & $0.4(0.3,0.7)$ & $0.7(0.5,1.0)$ \\
\hline T2D (n (\%)) & $16(19.75)$ & $57(70.37)$ & $4(4.94)$ & $4(4.94)$ & 81 \\
\hline HTN (n (\%)) & $42(16.73)$ & $162(64.54)$ & $42(16.73)$ & $5(1.99)$ & 251 \\
\hline CKD (n (\%)) & $16(23.19)$ & $44(63.77)$ & $8(I 1.59)$ & $\mathrm{I}(\mathrm{l} .45)$ & 69 \\
\hline \multicolumn{6}{|l|}{ Female } \\
\hline Number (\%) & $69(5.1)$ & $563(41.67)$ & $592(43.82)$ & $127(9.4)$ & $135 \mid$ \\
\hline HDL-C (IQR) (mmol/L) & $0.93(0.84,1.00)$ & $1.36(1.24,1.46)$ & $1.73(1.04,1.87)$ & $2.19(2.12,2.33)$ & $1.59(1.36,1.80)$ \\
\hline Age (IQR) (years) & $61(49,69)$ & $59(50,69)$ & $56(49,66)$ & $57(47,66)$ & $57(49,67)$ \\
\hline BMI (IQR) $\left(\mathrm{kg} / \mathrm{m}^{2}\right)$ & $26.0(23.6,28.2)$ & $24.8(22.8,27.7)$ & $23.2(21.2,25.2)$ & $22.4(20.5,25.0)$ & $23.9(21.7,26.4)$ \\
\hline FBG (IQR) (mmol/L) & $5.7(4.9,6.8)$ & $5.2(4.8,5.9)$ & $4.9(4.6,5.4)$ & $4.9(4.6,5.4)$ & $5.0(4.7,5.6)$ \\
\hline LDL-c (IQR) (mmol/L) & $2.1(1.6,2.5)$ & $3.1(2.5,3.8)$ & $3.3(2.8,4.0)$ & $3.5(2.9,4.2)$ & $3.2(2.6,3.9)$ \\
\hline TG (IQR) (mmol/L) & $3.4(2.1,5.5)$ & I.6(I.I,2.2) & I.I $(0.9, I .4)$ & $1.0(0.8,1.2)$ & $1.2(1.0,1.8)$ \\
\hline $\mathrm{TC}(\mathrm{IQR})(\mathrm{mmol} / \mathrm{L})$ & $4.8(4.1,5.5)$ & $5.2(4.6,6.0)$ & $5.6(5.0,6.4)$ & $6.2(5.5,7.1)$ & $5.4(4.8,6.3)$ \\
\hline VLDL-c(IQR) (mmol/L) & I.5(I.0,2.4) & $0.7(0.5,1.0)$ & $0.5(0.4,0.6)$ & $0.4(0.4,0.5)$ & $0.6(0.4,0.8)$ \\
\hline T2D (n (\%)) & II (9.64) & $6 I(53.5 I)$ & $34(29.82)$ & $8(7.02)$ & 114 \\
\hline HTN (n (\%)) & $24(6.54)$ & $195(53.13)$ & $125(34.06)$ & $23(6.27)$ & 367 \\
\hline CKD (n (\%)) & $6(6.45)$ & $52(55.91)$ & $26(27.96)$ & $9(9.68)$ & 93 \\
\hline
\end{tabular}

Abbreviations: HDL-c, high-density lipoprotein cholesterol; L-HDL-c, low HDL-c; M-HDL-c, medium HDL-c; H-HDL-c, high HDL-c; E-HDL-c, extremely high HDL-c; BMI, body mass index; FBG, fasting blood glucose; LDL-c, low-density lipoprotein cholesterol; TG, triglyceride; TC, total cholesterol; VLDL-c, very-low-density lipoprotein cholesterol; T2D, type 2 diabetes mellitus; HTN, hypertension; CKD, chronic kidney disease.

Nevertheless, L-HDL-c and M-HDL-c still accounted for $81.2 \%$ of male patients with HTN, although no statistical significance was found (Figure 2E).

\section{Correlation Between HDL-c and Inflammatory Indicators}

In both sexes, the HsCRP level generally declined as the HDLc level increased in the three diseases (Figure 3A, 3B, 3C). However, trend divergence was seen in females and males for the IL-6 concentration. In males, the IL-6 level was inversely correlated with the HDL-c level, whereas in females, the IL-6 level decreased to the lowest level in the H-HDL-c group and increased again in the E-HDL-c group (Figure 3D, 3E, 3F).

\section{Correlation Between HDL-c and Early- Stage Renal Impairment Indicators}

No distinct correlation was observed among U-NAG, Cys-C and HDL-c levels in females (Figure 4A, 4B, $4 \mathrm{C}, 4 \mathrm{G}, 4 \mathrm{H}, 4 \mathrm{I})$. In males, however, the Cys-C level decreased significantly with increasing HDL-c levels in patients with T2D $(p<0.05$, Figure $4 \mathrm{G})$. Moreover, the RBP level was also negatively correlated with the HDLc level among all patients from the L-HDL-c to the H-HDL-c level but increased as the HDL-c level continued to rise, which was significant in patients with HTN $(p<0.05$, Figure 4E) and in female patients with T2D $(p<0.05$, Figure 4D).

\section{Differences in the Levels of HDL-c, Inflammation and Early-Stage Renal Impairment Indicators Between Females and Males}

Among the three diseases, a universally higher level of HDL-c was detected in females than in males (Table 2). Concordant with this finding, lower levels of the renal impairment indicators U-NAG, RBP and Cys-C were observed in females (Table 2, Figure 4), but the levels of 

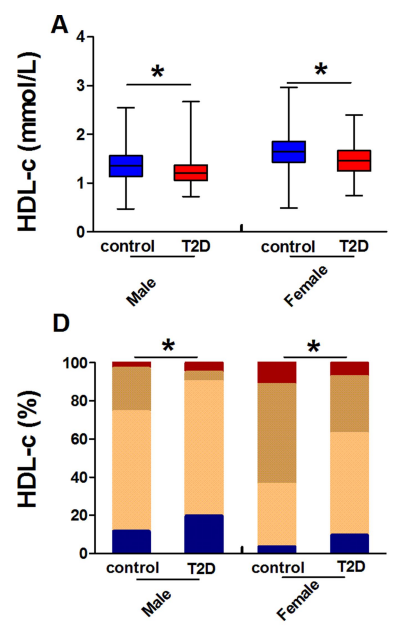

B

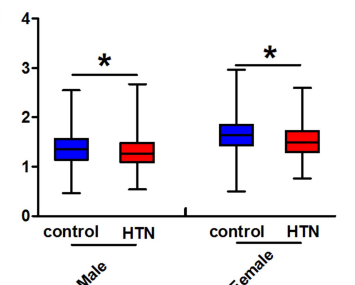

E

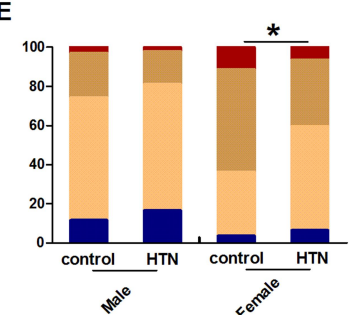

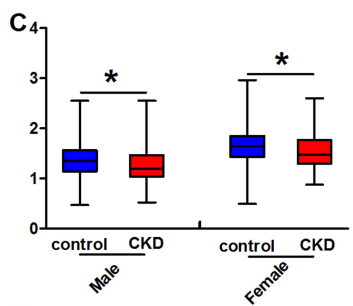

F

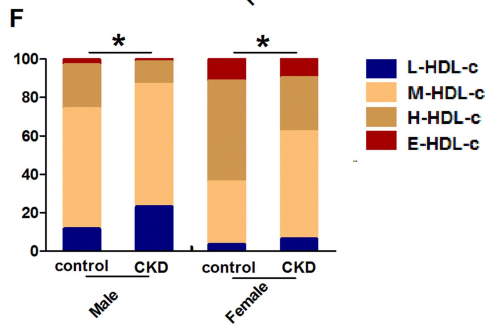

Figure 2 Comparison of HDL-c between patients with T2D, HTN, and CKD and the controls in males and females. The comparison of HDL-c in patients with T2D and controls (A), with HTN and controls (B), with CKD and controls (C). Comparison of the HDL-c distribution ratio in patients with T2D and the controls (D), with HTN and the controls $(\mathbf{E})$, with CKD and the controls $(\mathbf{F})$. *Indicates $p<0.05$.

Abbreviations: T2D, type 2 diabetes mellitus; HTN, hypertension; CKD, chronic kidney disease; HDL-c, high-density lipoprotein cholesterol; control, healthy participants; L-HDL-c, low HDL-c; M-HDL-c, medium HDL-c; H-HDL-c, high HDL-c; E-HDL-c, extremely high HDL-c.

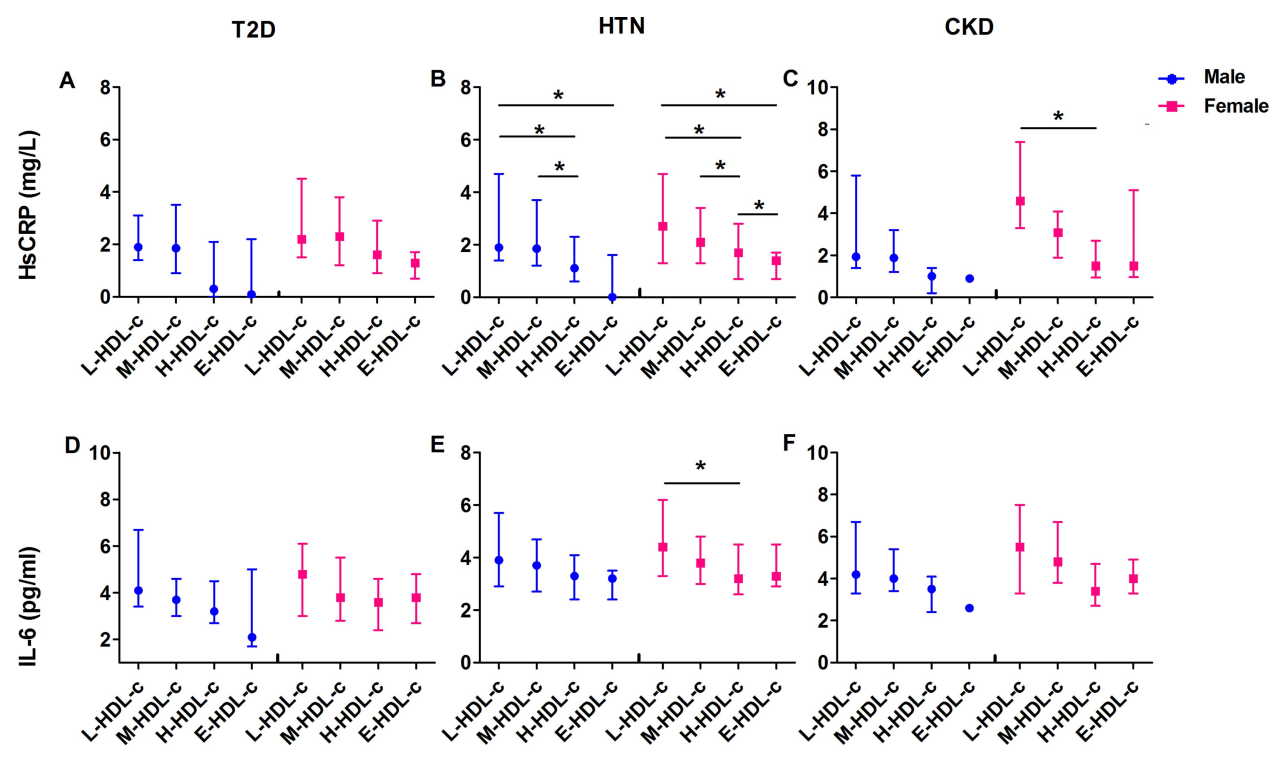

Figure 3 The correlation between HsCRP, IL-6 and HDL-c in male and female patients with T2D, HTN, or CKD. The correlation between HsCRP and HDL-c in patients with T2D (A), patients with HTN (B), and patients with CKD (C). The correlation between IL-6 and HDL-c in patients with T2D (D)), patients with HTN (E), and patients with CKD (F). *Indicates $p<0.05$.

Abbreviations: HsCRP, high-sensitivity C-reactive protein; IL-6, interleukin-6; T2D, type 2 diabetes mellitus; HTN, hypertension; CKD, chronic kidney disease; HDL-c, high-density lipoprotein cholesterol; control, healthy participants; L-HDL-c, low HDL-c; M-HDL-c, medium HDL-c; H-HDL-c, high HDL-c; E-HDL-c, extremely high HDL-c.

HsCRP and IL-6 in females were higher than those in males (Table 2, Figure 3).

\section{Discussion}

In this study, we found that the HDL-c levels of patients with T2D, HTN and CKD were all lower than those of the control group and more distributed in the L-HDL-c and M-HDL-c levels in both sexes. Moreover, there was not only a sex difference in the correlation between the HDL level and HsCRP, IL-6, U-NAG, RBP and Cys-C levels but also a discrepancy within a disease entity in the same sex concerning the relation between the HDL-c level and these variables, especially in HTN.

First, differential levels of HDL-c between patients with T2D, HTN or CKD and the control were found. Our results are consistent with most other studies. ${ }^{28-30}$ HDL-c was constantly independently and inversely related to the risks of hyperglycemia, hypertension and hyperuricemia. Therefore, 
T2D
HTN
CKD
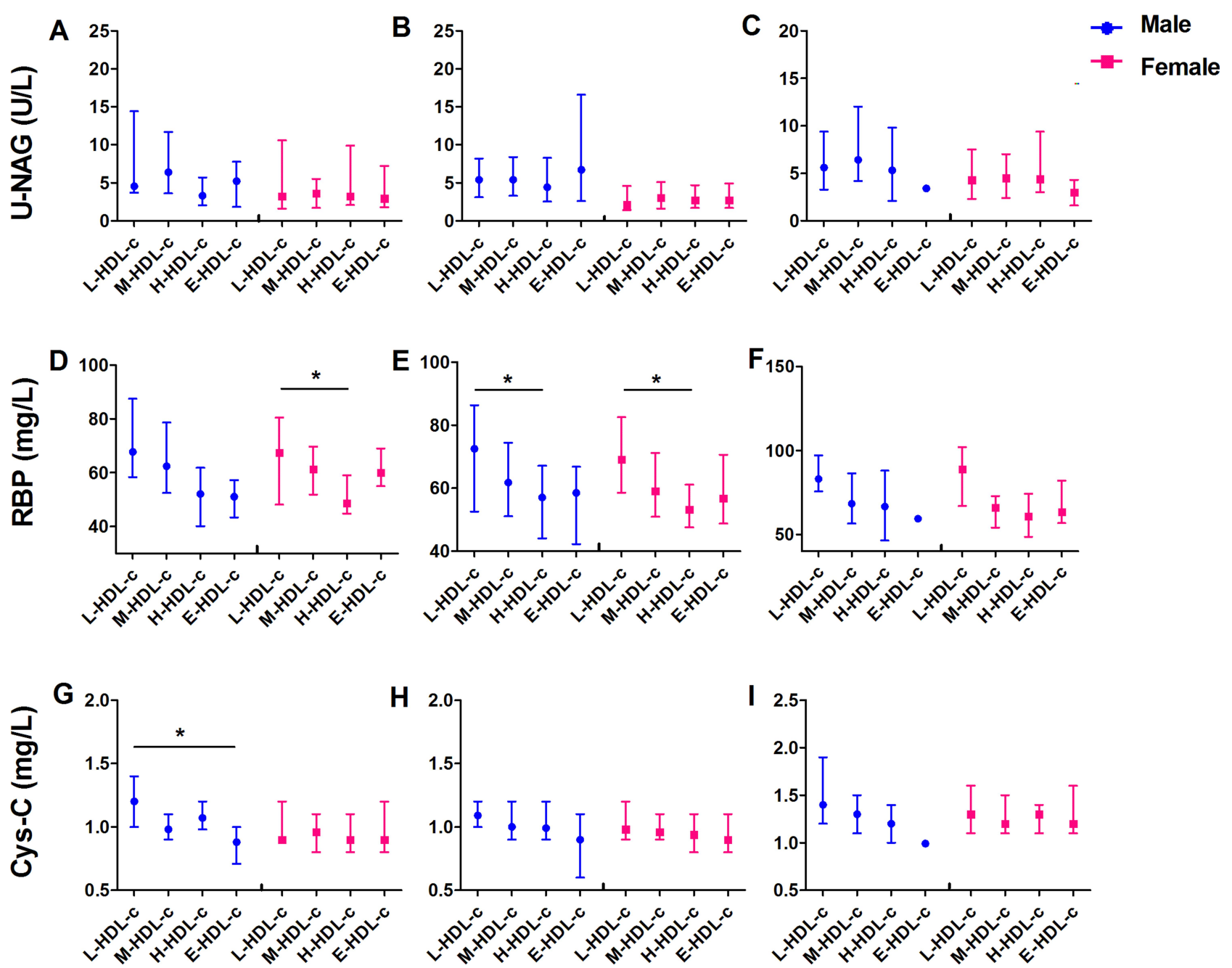

Figure 4 The correlation between U-NAG, RBP, Cys-C and HDL-c in male and female patients with T2D, HTN, or CKD. The correlation between U-NAG and HDL-c in patients with T2D (A), patients with HTN (B), and patients with CKD (C). The correlation between RBP and HDL-c in patients with T2D (D), patients with HTN (E), and patients with CKD (F). The correlation between Cys-C and HDL-c in patients with T2D (G), patients with HTN (H), and patients with CKD (I). *Indicates $p<0.05$. Abbreviations: U-NAG, urine $n$-acetyl- $\beta$-d-glucosidase; RBP, retinol binding protein; Cys-C, cystatin c; T2D, type 2 diabetes mellitus; HTN, hypertension; CKD, chronic kidney disease; HDL-c, high-density lipoprotein cholesterol; control, healthy participants; L-HDL-c, low HDL-c; M-HDL-c, medium HDL-c; H-HDL-c, high HDL-c; E-HDL-c, extremely high HDL-c.

an insufficient HDL-c volume in patients with dyslipidemiaassociated diseases became the basis for clinical attempts to treat patients with dyslipidemia by increasing the level of HDL-c. However, an effort to increase HDL-c did not yield the expected clinical effect, suggesting that further analysis of the relationship between HDL-c and related diseases, such as the sex differences and disease-related distribution differences of HDL-c in patients with dyslipidemia, is needed.

Next, we found intriguing sex differences when viewing the index values. The HDL-c level in females was higher overall than that in males regardless of T2D, HTN or CKD. Regarding U-NAG, RBP and Cys-C, indicators reflecting early-stage renal injury, we found reasonably lower values in females. Nonetheless, comparison of HsCRP and IL-6, indicators of the proinflammatory response, showed that indicator levels in females were universally higher than those in males. In addition, HDLc differences among patients with different diseases also deserve to be investigated. HsCRP, IL-6, and RBP were the most significantly correlated with HDL-c levels in patients with HTN $(p<0.05)$, yet we failed to find such correlations in patients with T2D and CKD. HDL particles exert a variety of effects, such as anti-inflammation, antioxidation, antithrombosis and antiapoptosis. ${ }^{31}$ Overall, HDL-c manifests as impeding the progression of inflammation and renal impairment. However, our findings 
Table 2 Comparison of HDL-c, Inflammatory and Early-Stage Renal Impairment Indicators in Patients with T2D, HTN or CKD and the Controls (Healthy Participants)

\begin{tabular}{|c|c|c|c|c|c|c|}
\hline & \multicolumn{3}{|l|}{ Male } & \multicolumn{3}{|l|}{ Female } \\
\hline & Control & T2D & $p$ & Control & T2D & $p$ \\
\hline HDL-c (IQR) (mmol/L) & I.4(I.I,I.6) & $1.2(I .1,1.4)$ & 0.000 & $1.6(1.4,1.9)$ & $1.5(1.3,1.7)$ & 0.000 \\
\hline L-HDL-c (n (\%)) & $44(I I .7)$ & $16(19.8)$ & 0.000 & $28(3.6)$ & $\mathrm{II}(9.6)$ & 0.000 \\
\hline M-HDL-c (n (\%)) & $235(62.7)$ & $57(70.4)$ & & $255(32.8)$ & $6 I(53.5)$ & \\
\hline H-HDL-c (n (\%)) & $85(22.7)$ & $4(4.9)$ & & $407(52.4)$ & $34(29.8)$ & \\
\hline E-HDL-c (n (\%)) & $\mathrm{II}(2.9)$ & $4(4.9)$ & & $87(11.2)$ & $8(7.0)$ & \\
\hline Total (n (\%)) & $375(100)$ & $81(100)$ & & $777(100)$ & $114(100)$ & \\
\hline HsCRP (IQR) (mg/L) & I.2(0.4,2.4) & $1.8(0.8,3.0)$ & 0.008 & $1.2(0.0,2.2)$ & $1.8(I . I, 3.4)$ & 0.000 \\
\hline IL-6 (IQR) (pg/mL) & $3.2(2.4,4.4)$ & $3.7(2.9,4.7)$ & 0.037 & $2.9(2.4,3.7)$ & $3.7(2.8,5.0)$ & 0.000 \\
\hline U-NAG (IQR) (U/L) & $3.5(2.1,5.9)$ & $5.5(3.4,10.7)$ & 0.000 & $2 . I(1.2,3.6)$ & $3.6(1.8,6.9)$ & 0.000 \\
\hline RBP (IQR) (mg/L) & $58.0(50.1,68.6)$ & $61.9(51.8,78.4)$ & 0.019 & $49.2(41.0,57.7)$ & $57.8(48.8,68.1)$ & 0.000 \\
\hline \multirow[t]{2}{*}{ Cys-C (IQR) (mg/L) } & $0.9(0.9,1.0)$ & $1.0(0.9,1.2)$ & 0.000 & $0.8(0.8,0.9)$ & $0.9(0.8,1.1)$ & 0.000 \\
\hline & Control & HTN & $p$ & Control & HTN & $p$ \\
\hline HDL-c (IQR) (mmol/L) & $1.4(1.1,1.6)$ & $1.27(1.1,1.5)$ & 0.002 & $1.6(1.4,1.9)$ & $1.5(1.3,1.7)$ & 0.000 \\
\hline L-HDL-c (n (\%)) & $44(I I .7)$ & $42(16.7)$ & 0.114 & $28(3.6)$ & $24(6.5)$ & 0.000 \\
\hline M-HDL-c (n (\%)) & $235(62.7)$ & $162(64.5)$ & & $255(32.8)$ & $195(53.1)$ & \\
\hline H-HDL-c (n (\%)) & $85(22.7)$ & $42(16.7)$ & & $407(52.4)$ & $125(34.1)$ & \\
\hline E-HDL-c (n (\%)) & $\mathrm{II}(2.9)$ & $5(1.9)$ & & $87(11.2)$ & $23(6.3)$ & \\
\hline Total (n (\%)) & $375(100)$ & $25 I(100)$ & & $777(100)$ & $367(100)$ & \\
\hline HsCRP (IQR) (mg/L) & I.2(0.4,2.4) & $1.7(1.1,3.2)$ & 0.000 & $1.2(0.0,2.2)$ & $1.9(1.1,3.1)$ & 0.000 \\
\hline IL-6 (IQR) (pg/mL) & $3.2(2.4,4.4)$ & $3.6(2.7,4.7)$ & 0.025 & $2.9(2.4,3.7)$ & $3.6(2.8,4.7)$ & 0.000 \\
\hline U-NAG (IQR) (U/L) & $3.5(2.1,5.9)$ & $5.3(3.2,8.3)$ & 0.000 & $2.1(1.2,3.6)$ & $2.9(1.6,4.9)$ & 0.000 \\
\hline RBP (IQR) (mg/L) & $58.0(50.1,68.6)$ & $61.4(50.5,74.2)$ & 0.009 & $49.2(41.0,57.7)$ & $57.5(49.3,69.2)$ & 0.000 \\
\hline \multirow[t]{2}{*}{ Cys-C (IQR) (mg/L) } & $0.9(0.9,1.0)$ & $1.0(0.9,1.2)$ & 0.000 & $0.8(0.8,0.9)$ & $0.9(0.9,1.1)$ & 0.000 \\
\hline & Control & CKD & $p$ & Control & CKD & $p$ \\
\hline HDL-c (IQR) (mmol/L) & I.4(I.I,I.6) & $1.19(1.0,1.5)$ & 0.001 & $1.6(1.4,1.9)$ & $1.5(1.3,1.8)$ & 0.001 \\
\hline L-HDL-c (n (\%)) & $44(I I .7)$ & $16(23.2)$ & 0.025 & $28(3.6)$ & $6(6.5)$ & 0.000 \\
\hline M-HDL-c (n (\%)) & $235(62.7)$ & $44(63.8)$ & & $255(32.8)$ & $52(55.9)$ & \\
\hline H-HDL-c (n (\%)) & $85(22.7)$ & $8(11.6)$ & & $407(52.4)$ & $26(28.0)$ & \\
\hline E-HDL-c (n (\%)) & $\mathrm{II}(2.9)$ & $\mathrm{I}(\mathrm{I} .4)$ & & $87(11.2)$ & $9(9.7)$ & \\
\hline Total (n (\%)) & $375(100)$ & $69(100)$ & & $777(100)$ & $93(100)$ & \\
\hline HsCRP (IQR) (mg/L) & I.2(0.4,2.4) & I.7(I.I,3.I) & 0.002 & $1.2(0.0,2.2)$ & $2.5(1.4,3.9)$ & 0.000 \\
\hline IL-6 (IQR) (pg/mL) & $3.2(2.4,4.4)$ & $3.9(3.3,5.5)$ & 0.000 & $2.9(2.4,3.7)$ & $4.5(3.4,6.2)$ & 0.000 \\
\hline U-NAG (IQR) (U/L) & $3.5(2.1,5.9)$ & $6.1(3.7,10.9)$ & 0.000 & $2.1(1.2,3.6)$ & $4.2(2.5,7.3)$ & 0.000 \\
\hline RBP (IQR) (mg/L) & $58.0(50.1,68.6)$ & $73.2(57.3,88.7)$ & 0.000 & $49.2(41.0,57.7)$ & $64.3(53.9,75.1)$ & 0.000 \\
\hline Cys-C (IQR) (mg/L) & $0.9(0.9,1.0)$ & $1.3(1.1,1.5)$ & 0.000 & $0.8(0.8,0.9)$ & $1.2(1.1,1.5)$ & 0.000 \\
\hline
\end{tabular}

Abbreviations: T2D, type 2 diabetes mellitus; HTN, hypertension; CKD, chronic kidney disease; control, the healthy participants; HDL-c, high-density lipoprotein cholesterol; L-HDL-c, low HDL-c; M-HDL-c, medium HDL-c; H-HDL-c, high HDL-c; E-HDL-c, extremely high HDL-c; HsCRP, high-sensitivity C-reactive protein; IL-6, interleukin-6; U-NAG, urine n-acetyl- $\beta$-d-glucosidase; RBP, retinol binding protein; Cys-C, cystatin c.

indicated that the anti-inflammation and renal protection of HDL-c were not completely parallel to its concentration and that HDL particles likely offer heterogeneous functions in the different sexes and different diseases. HDL-c is a complicated large molecule composed of many proteins and lipids, with apolipoprotein AI (Apo AI) occupying more than $70 \%$ of the total protein mass. It has been shown that when ApoAI is replaced by Apo-AII, ${ }^{32}$ HDL-c is altered to a smaller molecule and exerts an anti-inflammatory effect with the potential participation of HDLassociated miRNA. ${ }^{33}$ Various associated changes in miRNAs, proteins, and lipids determine numerous patterns of the HDL submicron structure, which provides heterogeneous functions within a particular disease setting. To 
expand the benefit of HDL-c-raising therapy, further studies on the components and properties of the HDL-c complex in different diseases are needed. Since the proinflammatory indicators were not lower in females in line with their higher HDL-c levels, in current clinical practice, barely assessing the efficacy of HDL-c treatment via measurement of HDL-c concentration seems inadequate. To better reflect the anti-inflammatory effect of HDL-c, it is feasible to apply additional monitoring indicators, such as hsCRP and IL-6. Some studies found that low serum HDL-c concentrations were negatively correlated with hsCRP and IL- $6 .{ }^{34}$ Further research showed that HDL-c exerts its anti-inflammatory properties by preventing LPS from activating the Toll-like receptor 4 (TLR4) signaling pathway in monocytes to reduce the expression of the proinflammatory cytokine IL-6, which subsequently stimulates the secretion of CRP. ${ }^{35}$ According to our study, HsCRP levels generally declined as HDL-c levels increased in patients with T2D, HTN and CKD. IL-6 was decreased to its lowest level in the E-HDL-c group in males but in the H-HDL-c group in females. Therefore, in order to achieve the maximum antagonistic effect on chronic inflammation, it seems reasonable to control the HDL-C level in the range of $1.56-2.05 \mathrm{mmol} / \mathrm{L}$ in women, while higher levels of HDL-C may benefit men. Great awareness of the sex differences in HDL-c needs to be raised.

Finally, we evaluated the correlation between the indexes of early-stage renal impairment and HDL-c levels in three disease groups, which has rarely been investigated at present. In this study, U-NAG and Cys-C are both sensitive markers of glomerular filtration, but a significant correlation was unable to be observed between U-NAG and Cys-C levels and the HDL-c level. Fortunately, an inverse correlation between the RBP level and HDL-c level was seen in all three diseases and was significant in HTN. For both female and male patients with HTN, the RBP level decreased to the valley at the H-HDL-c level and climbed in the E-HDL-c group. This finding implied that extremely high levels of HDL-c did not play the expected protective role in renal injury, which is in alignment with the result of Ziyad Al-Aly et al. ${ }^{7}$ Therefore, in order to avoid adverse effects on the kidney when using HDL-c-elevating therapy, it may be appropriate to keep HDL-C in the range of H-HDL-c. The heterogeneity of HDL-c among T2D, HTN and CKD seems like it cannot be neglected.
In accordance with the collected data, the vast majority of the patients fell within the L-HDL-c and M-HDL-c groups (Figure 2D, 2E, 2F). Properly increasing the HDL$\mathrm{c}$ value might therefore provide a considerable benefit to the health of the population. Consequently, in addition to its level, taking the heterogeneous properties of HDL-c into account, physicians are supposed to create a more rational plan adjusted to a particular disease and sex. Furthermore, most studies of HDL-c currently focus on its impact on the final clinical outcome but fail to monitor its therapeutic efficacy and evaluate the rationality of the target value. This study found that HDL-c levels were significantly correlated with HsCRP and RBP levels in patients with HTN, and this correlation was completely consistent in males and females. HsCRP and RBP can be used as indicators to monitor the efficacy of HDL-c-raising therapy in HTN, which may enable patients to benefit from HDL-c treatment to a greater extent, thereby delaying disease progression and even improving the clinical outcome. However, the practical effect of the two indicators still needs to be confirmed by a large sample of multicenter studies.

\section{Conclusion}

By applying a detailed grouping of the patients in the current study, we noticed the existence of substantial differences in HDL-c levels between different types of disease and sex, and highlighted that a higher HDL level does not always predict a better clinical outcome of patients. Moreover, we found that both HsCRP and RBP correlated negatively with HDL-c in HTN patients, indicating that monitoring HsCRP and RBP may serve as indicators for therapeutic efficacy of HDL-c-raising medications in HTN patients, but not in T2D and CKD patients.

In the future, we will have deeper insight into the molecular composition and structure of HDL-c particles with the invention of convenient and high-throughput detection technology. On the basis of that possibility, the precise correlation between its function and submicron structure can be elaborated to provide a more accurate and rational guide for curing a series of diseases associated with lipid metabolism disorders.

\section{Ethics Approval}

The experimental scheme was approved by the ethics committee of the Third Affiliated Hospital of Southern Medical University $(201,708,011)$. 


\section{Acknowledgments}

The authors would like to thank all patients who participated in this study and all the community workers for their excellent secretarial assistance. In addition, they thank all the investigators, including Aiqun Liu, Peichun Gao, Yunyin Chen, Ziliang Zou, Haishan Chen, Xinyan Yang, Qin Zhou, Jing Ning, in the clinical laboratory of the Third Affiliated Hospital of Southern Medical University.

\section{Funding}

This work was supported by the Guangdong Provincial Key Laboratory Construction Project of China (grant number 2014B030301044), the Guangdong Provincial Natural Science Foundation (grant number 2020A1515011258), the Science and Technology Program of Guangzhou (NO.201604020015, No.201904010382), and the South Wisdom Valley Innovative Research Team Program (grant number CXTD-001).

\section{Disclosure}

The authors report no conflicts of interest in this work.

\section{References}

1. American Diabetes Association. Dyslipidemia management in adults with diabetes. Position statement \& ADA statement. Diabetes Care. 2004;27(suppl):S68-71. doi:10.2337/diacare.27.2007.S68

2. Kyung-Hyun C, Hye-Jeong P, Jae-Ryong K. Decrease in Serum HDL$\mathrm{C}$ level is associated with elevation of blood pressure: correlation analysis from the korean national health and nutrition examination survey 2017. Int J Environ Res Public Health. 2020; 17: undefined.

3. Tsuruya K, Yoshida H, Nagata M, et al. Impact of the triglycerides to high-density lipoprotein cholesterol ratio on the incidence and progression of CKD: A longitudinal study in a large Japanesepopulation. Am J Kidney Dis. 2015;66(6):972-983. doi:10.1053/j.ajkd.2015.05.011

4. Rosenson RS, Davidson MH, Le NA, Burkle J, Pourfarzib R. Underappreciated opportunities for high-density lipoprotein particles in risk stratification and potential targets of therapy. Cardiovasc Drug Ther. 2015;29:41-50. doi:10.1007/s10557-014-6567-0

5. Kontush A, Chapman MJ. Antiatherogenic small, dense HDL - guardian angel of the arterial wall?. Nat Clin Pract Cardiovasc Med. 2006;3:144-153.

6. Lincoff AM, Nicholls SJ, Riesmeyer JS, et al. Evacetrapib and cardiovascular outcomes in high-risk vascular disease. $N$ Engl J Med. 2017;376:1933-1942. doi:10.1056/NEJMoa1609581

7. Benjamin B, Yan X, Hong X, Sumitra B, Ziyad -A-A. Low levels of high-density lipoprotein cholesterol increase the risk of incident kidney disease and its progression. Kidney Int. 2016;89:886-896. doi:10.1016/j.kint.2015.12.034

8. Hirata A, Okamura T, Sugiyama D, et al. The relationship between very high levels of serum high-density lipoprotein cholesterol and cause-specific mortality in a 20 -year follow-up study of Japanese general population. $J$ Atheroscler Thromb. 2016;23:800-809. doi:10.5551/jat.33449

9. Madsen CM, Varbo A, Nordestgaard BG. Extreme high high-density lipoprotein cholesterol is paradoxically associated with high mortality in men and women: two prospective cohort studies. Eur Heart J. 2017;38:2478-2486. doi:10.1093/eurheartj/ehx163
10. Hirata A, Sugiyama D, Watanabe M, et al. Association of extremely high levels of high-density lipoprotein cholesterol with cardiovascular mortality in a pooled analysis of 9 cohort studies including 43,407 individuals: the EPOCH-JAPAN study. J Clin Lipidol. 2018;12:674684.e5. doi:10.1016/j.jacl.2018.01.014

11. Schunkert H, Konig IR, Kathiresan S, et al. Large-scale association analysis identifies 13 new susceptibility loci for coronary artery disease. Nat Genet. 2011;43:333-338. doi:10.1038/ng.784

12. Teslovich TM, Musunuru K, Smith AV, et al. Biological, clinical and population relevance of 95 loci for blood lipids. Nature. 2010;466:707-713. doi:10.1038/nature09270

13. Vitali C, Khetarpal SA, Rader DJ. HDL cholesterol metabolism and the risk of CHD: new insights from human genetics. Curr Cardiol Rep. 2017;19:132. doi:10.1007/s11886-017-0940-0

14. Kajani S, Sean C, Fiona C, Gillicuddy M. Unravelling HDL-looking beyond the cholesterol surface to the quality within. Int J Mol Sci. 2018;19:1971-1999.

15. Qaddoumi Mohammad G, Muath A, Hammad Maha M, et al. Investigating the role of myeloperoxidase and angiopoietin-like protein 6 in obesity and diabetes. Sci Rep. 2020;10(1):6170-6181. doi:10.1038/s41598-020-63149-7

16. Ghazi L, Baker Jason V, Sharma S, et al. Role of inflammatory biomarkers in the prevalence and incidence of hypertension among hiv-positive participants in the START Trial. Am $J$ Hypertension. 2020;33(1):43-52. doi:10.1093/ajh/hpz132

17. Jianhua W, Naifeng G, Chen Xiaolan et al. Coexistence of microinflammatory and macrophage phenotype abnormalities in chronic kidney disease. Int J Clin Exp Pathol. 2020;13:317-323.

18. World Health Organization. Definition and diagnosis of diabetes mellitus and intermediate hyperglycemia: report of a WHO/IDF consultation, 2006[M]. Geneva: WHO Document Production Services, 2006.

19. Chalmers J, MacMahon S, Mancia G, et al. WHO-ISH hypertension guidelines committee. 1999 world health organization-international society of hypertension guidelines for the management of hypertension. J Hypertens. 1999; 17:151-183.

20. Levey AS, Eckardt KU, Tsukamoto Y, et al. Definition and classification of chronic kidney disease: A position statement from Kidney Disease: improving Global Outcomes (KDIGO). Kidney Int. 2005;67 (6):2089-2100. doi:10.1111/j.1523-1755.2005.00365.x

21. Eknoyan G, Levin N. NKF-K/DOQI clinical practice guidelines: update 2000. Foreword. Am J Kidney Dis. 2001;37(1 Suppl):S5-S6. doi:10.1016/S0272-6386(01)70004-2

22. Moriyama Y, Okamura T, Inazu A, et al. A low prevalence of coronary heart disease among subjects with increased high-density lipoprotein cholesterol levels, including those with plasma cholesteryl ester transfer protein deficiency. Prev Med. 1998;27:659-667. doi:10.1006/pmed.1998.0340

23. Wang L, Gao P, Zhang M, et al. Prevalence and ethnic pattern of diabetes and prediabetes in China in 2013. JAMA. 2017;317 (24):2515-2523. doi:10.1001/jama.2017.7596

24. Wang Z, Chen Z, Zhang L, et al. Status of hypertension in china: results from the china hypertension survey, 2012-2015. Circulation. 2018;137 (22):2344-2356. doi:10.1161/CIRCULATIONAHA.117.032380

25. Ene-Iordache B, Perico N, Bikbov B, et al. Chronic kidney disease and cardiovascular risk in six regions of the world (ISN-KDDC): a cross-sectional study. Lancet Glob Health. 2016;4(5):e307-e319. doi:10.1016/S2214-109X(16)00071-1

26. Zhang L, Wang F, Wang L, et al. Prevalence of chronic kidney disease in China: a cross-sectional survey. Lancet. 2012;379 (9818):815-822. doi:10.1016/S0140-6736(12)60033-6

27. Taal MW. Chronic kidnev disease: towards a Iisk-based approach. Clin Med. 2016;16(Suppl 6):s117-s120.

28. Wang YL, Koh WP, Talaei M, Yuan JM, Pan A. Association between the ratio of triglyceride to high-density lipoprotein cholesterol and incident type 2 diabetes in Singapore Chinese men and women. $J$ Diab. 2017;9(7):689-698. doi:10.1111/1753-0407.12477 
29. Kunutsor SK, Kieneker LM, Bakker SJL, James RW, Dullaart RPF. The inverse association of HDL-cholesterol with future risk of hypertension is not modified by its antioxidant constituent, paraoxonase-1: the PREVEND prospective cohort study. Atherosclerosis. 2017;263:219-226. doi:10.1016/j.atherosclerosis. 2017.06.353

30. Ain QU, Asif N, Alam A, Gilani M, Noreen SW. Triglycerides-tohdlc ratio as a marker of cardiac disease and vascular risk factors in adults. J Coll Physicians Surg Pak. 2019;29(11):1034-1037. doi:10.29271/jcpsp.2019.11.1034

31. Camont L, Chapman MJ, Kontush A. Biological activities of HDL subpopulations and their relevance to cardiovascular disease. Trends Mol Med. 2011;17:594-603. doi:10.1016/j.molmed.2011.05.013
32. Kontush A, Lhomme M, Chapman MJ. Unraveling the complexities of the HDL lipidome. J Lipid Res. 2013;54:2950-2963.

33. Kajani S, Curley S, McGillicudd. FC. Unravelling HDL-looking beyond the cholesterol surface to the quality within. Int $J \mathrm{Mol} \mathrm{Sci}$. 2018;19:1971. doi:10.3390/ijms19071971

34. Wan Ahmad Wan NH, Sakri F, Mokhsin A, et al. Low serum high density lipoprotein cholesterol concentration is an independent predictor for enhanced inflammation and endothelial activation. PLoS One. 2015;10:e116867. doi:10.1371/journal.pone.0116867

35. Gillard BK, Lin HY, Massey JB, Pownall HJ. Apolipoproteins A-I, A-II and $\mathrm{E}$ are independently distributed among intracellular and newly secreted HDL of human hepatoma cells. Biochim Biophy Acta. 2009;1791:1125-1132. doi:10.1016/j.bbalip.2009.07.004

\section{Publish your work in this journal}

Diabetes, Metabolic Syndrome and Obesity: Targets and Therapy is an international, peer-reviewed open-access journal committed to the rapid publication of the latest laboratory and clinical findings in the fields of diabetes, metabolic syndrome and obesity research. Original research, review, case reports, hypothesis formation, expert opinion and commentaries are all considered for publication. The manuscript management system is completely online and includes a very quick and fair peer-review system, which is all easy to use. Visit http://www.dovepress.com/testimonials.php to read real quotes from published authors.

Submit your manuscript here: https://www.dovepress.com/diabetes-metabolic-syndrome-and-obesity-targets-and-therapy-journal 